\title{
Le droit international et le contrôle des mercenaires et des compagnies militaires privées
}

International Law and the Control of Mercenaries and Private Military Companies

\section{Christopher Kinsey}

Traducteur : Nicolas Wuest-Famôse

\section{(Q) OpenEdition}

\section{Journals}

Édition électronique

URL : http://journals.openedition.org/conflits/981

DOI : $10.4000 /$ conflits. 981

ISSN : $1777-5345$

Éditeur :

CCLS - Centre d'études sur les conflits lilberté et sécurité, L'Harmattan

Édition imprimée

Date de publication : 1 décembre 2003

Pagination : $91-116$

ISBN : 2-7475-6065-1

ISSN : 1157-996X

Référence électronique

Christopher Kinsey, «Le droit international et le contrôle des mercenaires et des compagnies

militaires privées », Cultures \& Conflits [En ligne], 52 | hiver 2003, mis en ligne le 03 juillet 2004, consulté le 30 mars 2021. URL : http://journals.openedition.org/conflits/981 ; DOI : https://doi.org/10.4000/ conflits.981

Ce document a été généré automatiquement le 30 mars 2021.

Creative Commons License 


\title{
Le droit international et le contrôle des mercenaires et des compagnies militaires privées
}

\author{
International Law and the Control of Mercenaries and Private Military \\ Companies
}

Christopher Kinsey

Traduction : Nicolas Wuest-Famôse

Cet article ${ }^{1}$ a pour objectif d'examiner la manière dont le droit international peut être mobilisé en tant qu'agent de contrôle des mercenaires et des compagnies militaires privées. Les traités internationaux passés, en vue de contrôler l'activité des mercenaires, comprennent les Protocoles additionnels I et II de la Convention de Genève (1949), la Convention de l'Organisation à l'unité africaine (OUA) pour l'élimination des mercenaires en Afrique (1972) et la Convention internationale contre le recrutement, l'utilisation, le financement et l'instruction de mercenaires (1989). Ces traités régulent principalement les relations entre les Etats concernés par l'utilisation de la violence internationale privée comprise en termes de mercenariat. Ces traités sont loin d'être parfaits. En effet, ils sont le reflet de la tension internationale entre l'Occident et des Etats du Tiers Monde, notamment africains, sur ce que ces Etats analysent comme une volonté de l'Occident de tolérer des activités de mercenariat audelà de leurs frontières. Pareille tension était particulièrement évidente dans les années 1960 et 1970 dans cette Afrique post-coloniale, et avait forcé la communauté internationale à considérer d'un peu plus près les activités des mercenaires. Quoi qu'il en soit, la méfiance à l'égard des mercenaires ne s'est jamais traduite par une condamnation légale pleine et entière que ce soit dans le droit international ou dans les législations internes.

Cet état de fait s'explique par la façon dont les Etats africains ont compris, durant ces quarante dernières années, la souveraineté et leurs rapports avec les forces de mercenaires. Alors que la difficulté d'établir une définition a été largement débattue dans d'autres articles, ce texte s'intéresse à la relation entre les problèmes de définition 
et les problèmes politiques associés à la mauvaise volonté de certains de mettre un terme aux activités mercenaires. Holds Bashir énonce ainsi qu'«il est très difficile de définir ce qu'est un mercenaire. Cela s'explique par les différents sens que le mot a eu à différentes époques. Les différents sens que le mot a acquis à travers l'histoire dépendent de l'esprit de l'époque ${ }^{2} »$. Ce problème existe encore aujourd'hui. Les mercenaires ne sont-ils des mercenaires que lorsque l'actualité politique des Etats le permet comme cela se reflète dans les traités internationaux cités précédemment? Les traités ne prohibent pas l'usage de la violence privée internationale en tant que mercenariat. Au contraire, cette violence est autorisée pour autant qu'elle bénéficie aux Etats qui ont élaboré le traité en premier. Alors que les compagnies de sécurité, dont la MPRI (Military Professional Resources Incorporated), Group4 et Control Risk Group, sont décrites comme étant mercenaires par des organisations opposées à l'application de la violence privée dans les affaires des Etats ${ }^{3}$, les gouvernements - en particulier occidentaux - ont résisté à la tentation de les étiqueter comme mercenaires car leurs activités sont comprises comme répondant à des inquiétudes sécuritaires légitimes des Etats.

Il s'agira ici d'examiner les problèmes juridiques dus à la récente émergence dans les affaires de sécurité internationale des compagnies militaires privées. La première partie de cet article se consacre au débat juridique sur l'emploi des mercenaires en examinant en particulier la nature complexe des conventions qui tentent de dresser une définition claire du mercenariat. Il démontre comment la présente définition du mercenariat, qui a servi un dessein politique durant la décolonisation (et sert toujours), facilite l'exploitation par l'industrie privée de la sécurité de vides juridiques dans le droit international, qui leur permettent ainsi d'offrir leurs services à des chefs dont les Etats sont déliquescents. L'article se poursuit par l'exploration des arguments entourant l'étendue de la responsabilité des gouvernements dans l'interdiction faite aux citoyens de s'engager dans des activités de mercenariat. Plus récemment, des gouvernements - et particulièrement en Afrique - ont voulu inclure dans les conventions la responsabilité pénale individuelle ainsi que la responsabilité des Etats, rendant de la sorte possible la poursuite devant les tribunaux d'individus pas seulement pour le fait d'être mercenaires, mais aussi pour les actions illégales qu'ils auraient pu commettre lors de combats en tant que mercenaires. S'attaquer à cette question dans le droit international prendra du temps, d'autant plus que les principaux dirigeants ignorent totalement la direction future que prendra l'usage de la force armée. Jusque là, il est très improbable qu'ils agiront, tant pour développer le marché de la sécurité privée que pour le réduire ${ }^{4}$.

Modification du droit et montée en puissance du mercenariat depuis 1945

Le contrôle des activités de mercenariat est un problème de longue date qui marque la limite de l'analyse juridique moderne et de la jurisprudence. Parmi les premiers juristes chargés de ces affaires certains se sont attachés à la question de l'embauche de mercenaires, mais, compte tenu du rôle joué par les forces de mercenaires dans la formation des armées européennes, de telles questions ont été largement ignorées jusqu'au milieu du XIXème siècle. Il en va de même hors d'Europe. Aussi, suite à leur utilisation dans les conflits coloniaux et dans d'autres situations ayant servi les intérêts nationaux, leur disparition a été plus lente à supposer qu'elle se soit produite. La Légion étrangère française est par exemple une force mercenaire en ce sens qu'elle a été créée à l'origine pour protéger les possessions coloniales françaises ${ }^{5}$ et qu'elle existe toujours aujourd'hui. 
Avant 1945, l'intérêt porté aux mercenaires s'exprimait principalement au travers du développement du droit à la neutralité. Un pays qui acceptait que son territoire national puisse être utilisé pour recruter ou recenser des mercenaires était réputé soutenir un belligérant. Cette position avait toutes les chances d'intégrer un Etat neutre à un conflit dans lequel il n'avait aucun intérêt, en particulier par les actions de représailles menées par l'un des belligérants. Il en a résulté l'intégration dans la Convention de La Haye de 1907 de dispositions interdisant le recrutement de mercenaires sur le territoire national ${ }^{6}$. De telles obligations étaient limitées aux pays assurant l'ordre public sur leur propre territoire, et ne comprenaient pas la possibilité pour des ressortissants de franchir une frontière et de s'engager dans une armée d'un pays tiers, comme cela a par exemple été le cas des Brigades internationales lors de la guerre civile espagnole. Un certain nombre d'Etats ont introduit une législation propre pour renforcer leurs obligations internationales, alors que d'autres, moins nombreux, ont cherché à contrôler l'action de leurs ressortissants souhaitant s'engager dans des armées étrangères.

$\mathrm{Au}$ lendemain de la Seconde guerre mondiale, des changements significatifs ont été apportés au droit régulant l'usage de la force dans la politique internationale. Toujours est-il que ces modifications introduites dans la législation n'ont pas réglé la question de l'implication de mercenaires au combat. Compte tenu des nombreux conflits, y compris idéologiques entre les Etats-Unis et l'Union soviétique immédiatement après la guerre, l'introduction de règlements relatifs au contrôle des activités de mercenaires constituait une faible priorité pour la communauté internationale. Cette indifférence a changé dans les années 1960, au moment de la décolonisation. Cette période est marquée par un accroissement tant du nombre que des activités des mercenaires, en particulier en Afrique centrale. Des mercenaires comme par exemple Mad Mike Hore, Jacques Schramme et Bob Denard, se sont battus au Congo dans les années 1960. Le concept de mercenaire a alors pris une plus grande importance pour la communauté internationale, mais bien plus encore pour les pays africains directement affectés par la présence de mercenaires impliqués dans les guerres de libération. En dépit de cela, la réaction de la communauté internationale s'est limitée à la réaffirmation de l'existence de l'article $2 \S 4$ de la Charte des Nations unies ${ }^{7}$, en expliquant les implications logiques sous-tendues par cet article.

Il faudra attendre la fin des années 1960 pour que l'usage de mercenaires contre les mouvements de libération nationale se battant pour l'indépendance des territoires coloniaux soit déclaré criminel par la Résolution 2465 de l'Assemblée générale de $\mathrm{l}^{\prime} \mathrm{ONU}^{8}$, désignant ainsi les mercenaires comme hors-la-loi'. Cette position a été plus tard endossée par d'autres résolutions relatives au colonialisme. Ceci, comme l'explique Taulbee (nous y reviendrons), a marqué un changement significatif en dépassant le concept de responsabilité collective assuré par le droit international pour lui préférer celui de responsabilité pénale individuelle. Ces résolutions ont de plus exigé des Etats tiers de respecter le droit traditionnel interdisant le recrutement de mercenaires sur leur territoire national, et de faire entrer en vigueur des lois interdisant à leurs ressortissants de s'engager dans des activités mercenaires ${ }^{10}$.

La Convention de l'OUA, adoptée en 1972 sur l'élimination des mercenaires, reflète les tendances que l'on trouve dans les résolutions de l'ONU relatives aux mercenaires ${ }^{11}$. La Convention étend ainsi l'obligation de l'Etat sur le contrôle des activités de ses ressortissants, en rendant les Etats responsables de l'interdiction et du châtiment pour 
toute activité liée aux mercenaires et qui pourrait se produire dans leurs juridictions ${ }^{12}$. L'abandon de la position traditionnelle tenant les Etats pour individuellement responsables au profit d'une responsabilité collective est le résultat de la Convention de l'OUA. Les individus doivent soit remplir les conditions consignées à l'article $1^{13}$, qui définit ce qu'est un mercenaire, soit se conformer aux conditions qui concernent ceux qui recrutent ou assistent les mercenaires par l'entraînement, l'aide financière, ou la protection contre toute poursuite. En sus, on y trouve l'adoption par les Etats contractants d'une obligation d'empêcher leurs ressortissants de participer à des activités mercenaires telles que définies dans la Convention ${ }^{14}$. Ainsi, les Etats contractants se doivent de poursuivre dans leur juridiction, toute personne, quelle que soit sa nationalité, et qui est accusée d'activité mercenaire ${ }^{15}$. L'article 1 est rédigé ainsi ${ }^{16}$ :

$[A]$ «mercenaire " définit toute personne, qui n'est pas le ressortissant d'un Etat contre lequel ses actions sont dirigées, et qui est employé, enrôlé ou lié volontairement à une personne, à un groupe ou à une organisation dont l'objectif est de:

renverser par les armes ou par tout autre moyen le gouvernement d'un Etat membre de l'Organisation à l'unité africaine ;

de miner l'indépendance, l'intégrité territoriale ou l'activité normale des institutions du dit Etat ;

de bloquer par quelque moyen les activités de tout mouvement de libération nationale reconnu par l'Organisation à l'unité africaine.

Malgré toutes ces dispositions, le Convention de l'OUA n'interdit pas totalement le recrutement de mercenaires. Conformément à l'article 1, il est interdit aux gouvernements ou à tout autre groupe, d'employer des mercenaires dans l'intention de se défendre eux-mêmes d'actions d'un mouvement de libération nationale reconnu par l'OUA. L'objectif premier de cet article était d'abord de mettre un terme aux activités des mercenaires européens combattant au profit des gouvernements minoritaires de Rhodésie et d'Afrique du Sud. En revanche, les non-ressortissants qui n'entrent pas dans les catégories définies de mercenaires peuvent être employés par un gouvernement pour se défendre de groupes dissidents au sein de ses propres frontières, comme dans le cas des troupes cubaines qui ont combattu aux côtés du Mouvement populaire de libération de l'Angola (MPLA) durant la guerre civile angolaise. La raison manifeste est la possibilité d'autoriser les gouvernements africains à soutenir les mouvements de libération nationale, sans se donner les moyens nécessaires à l'émergence de tels mouvements dans leur propre pays et qui pourraient un jour opérer contre eux ${ }^{17}$. Concomitamment, les gouvernements africains voulaient empêcher toute possibilité aux mercenaires d'opérer contre ces mêmes mouvements de libération nationale.

La capture et le procès de treize mercenaires en Angola en 1976 ont une nouvelle fois porté l'attention internationale sur l'activité mercenaire. Tous étaient accusés du crime d'être mercenaire ${ }^{18}$. Sur les treize accusés, quatre ont été condamnés à mort et les autres à de lourdes peines de prison. Une des conséquences de ce procès a été la rédaction du projet de Convention de Luanda sur la prévention et la suppression des mercenaires (1976) ${ }^{19}$. Le projet de convention de Luanda insiste lui aussi sur la responsabilité individuelle des Etats pour empêcher leurs ressortissants de prendre part à des activités mercenaires, ou d'être eux-mêmes des mercenaires. La responsabilité des Etats en tant que telle est traitée à l'article $3^{20}$ car elle rend les 
responsables gouvernementaux susceptibles de poursuites pénales s'ils entreprennent d'employer, d'aider ou de recruter des mercenaires. Aussi, l'absence de poursuites par un Etat de tout responsable qui aurait entrepris de telles activités, crée selon Taulbee, "une responsabilité internationale de l'Etat fautif $»^{21}$. A l'article $5^{22}$ transparaît l'attitude des membres du Tribunal Révolutionnaire Populaire qui a présidé le procès, en un sens où " un mercenaire porte la responsabilité à la fois d'être un mercenaire et d'avoir ainsi commis des crimes ${ }^{23}$. L'article le plus important est certainement l'article $4^{24}$ qui prive les mercenaires du statut de combattant de plein droit. Les mercenaires capturés ne se sont ainsi pas vus conférer le statut protecteur de prisonnier de guerre. Ce qui, selon Hampson, "violait le principe d'égalité des belligérants et rendait plus confuse la séparation entrejus ad bellumetjus in bello $\aleph^{25}$. Cette disposition a été incorporée plus tard, en 1977, au Protocole additionnel de Genève ${ }^{26}$. Néanmoins, la définition de mercenaire, élaborée par les négociateurs de Genève est plus limitée dans la portée que celle exprimée dans le projet de Convention de Luanda. En même temps, le Protocole additionnel I autorise les Etats à conférer le statut de prisonnier de guerre aux mercenaires, si les Etats le souhaitent. Ce qui est clairement compris et traité dans le Protocole Additionnel $\mathrm{II}^{27}$, car le texte étend aux mercenaires le traitement humanitaire de base et les protections conférées habituellement aux personnes au pouvoir d'une partie au conflit, et qui autrement ne peuvent se voir attribuer un traitement plus favorable.

Une définition juridique

Comment devrions-nous alors définir le mercenaire? La notion traditionnelle de mercenaire est la suivante: "un soldat voulant vendre ses compétences militaires au plus offrant, et pour qui la cause importe peu ${ }^{28}$. De son côté Mockler pense que la marque réelle du mercenaire consiste en "la dévotion à la guerre dans son propre intérêt. Ainsi, le mercenaire peut être distingué du soldat professionnel dont la marque est généralement la dévotion aux signes extérieurs d'apparat de la profession militaire plutôt que le combat réel ${ }^{29}$. Bien que ces définitions générales soient exactes, elles ne donnent pas une définition suffisamment précise. Or c'est là une question essentielle dès lors que des individus se voient refuser des droits importants selon la catégorie dans laquelle ils peuvent tomber. De plus, comme la partie du droit international couvrant les activités mercenaires évolue, les Etats n'auront pas d'autre choix que de prendre en main l'obligation de contrôler ces activités déterminées par la portée de la définition.

La définition n'a finalement émergé, dans le Protocole additionnel I à l'article 47 de la Convention de Genève (1949), qu'une fois que les Etats ont été assurés du fait que leurs intérêts politiques étaient bien protégés. Ainsi, la définition tient-elle compte des différents intérêts étatiques devant trouver un équilibre entre la précision et la portée générale. Comme le dit Taulbee « il a fallu trouver un juste milieu entre le besoin de fournir des paramètres généraux d'évaluation contextuelle des éléments, et les conditions qui décrivent de façon rigoureuse et exhaustive les personnes, les situations et les activités ${ }^{30} »$. Une définition trop détaillée pourrait se révéler trop rigide et rendre ainsi impossible une adaptation à de nouvelles circonstances. D'un autre côté, une définition trop brève qui permettrait diverses interprétations dans son application laisserait ouverte la porte à des abus. Une définition bien trop générale permettrait des interprétations sujettes à des calculs politiques ou idéologiques. 
La définition en cours donnée par le droit international et consignée dans le Protocole additionnel I à l'article 47 de la Convention de Genève (1949) statut sur ce qu'est un mercenaire selon les critères suivants ${ }^{31}$ :

(a) il est spécialement recruté dans le pays ou à l'étranger pour combattre dans un conflit armé; (b) il prend de facto une part directe aux hostilités; (c) il prend part aux hostilités essentiellement en vue d'obtenir un avantage personnel et auquel est effectivement promis, par une partie en conflit ou en son nom, une rémunération matérielle nettement supérieure à celle qui est promise ou payée à des combattants ayant un rang et une fonction analogues dans les forces armées de cette partie; (d) il n'est ni ressortissant d'une partie en conflit, ni résident du territoire contrôlé par une partie au conflit; (e) il n'est pas membre des forces armées d'une partie en conflit; et (f) il n'a pas été envoyé par un Etat autre qu'une partie en conflit en mission officielle en tant que membre des forces armées dudit Etat.

La rédaction de la définition est ainsi faite qu'elle exclut les ressortissants étrangers membres de forces armées d'un pays tiers (comme cela a été le cas pour les personnes qui ont servi dans les Brigades internationales durant la guerre civile espagnole) et là où la communauté internationale est disposée à tolérer de telles personnes sans qu'elles ne tombent dans la définition du mercenaire. De plus, l'article $47 \mathrm{du}$ Protocole I ignore les personnels militaires étrangers intégrés aux forces armées d'un autre Etat. Ici, on pourrait y inclure la Légion étrangère française ou les Gurkhas. La définition laisse aussi en suspens le cas de ceux amenés à combattre par idéologie ${ }^{32}$ ou sympathie religieuse, et ceux qui n'auraient pas participé directement aux hostilités. Enfin, les étrangers employés comme conseillers militaires ou formateurs ne sont pas plus concernés par la définition.

Sans définition pertinente, le problème se pose de savoir comment s'assurer que les Etats se conforment au droit international dans le domaine du contrôle des mercenaires. Alors que les Etats ont accepté le principe (par l'adoption de résolutions de l'Assemblée générale) d'interdire tant par omission que par action à un groupe armé de lancer une invasion d'un Etat à partir de leur propre territoire, les Etats membres n'ont toutefois pu empêcher leurs ressortissants de rejoindre des groupes de mercenaires. Devant cet aveu d'échec, la communauté internationale a reconnu la nécessité d'une convention internationale. Durant la trente-cinquième session de l'Assemblée générale, il a été décidé de rédiger un projet de Convention internationale contre le recrutement, l'utilisation, le financement et l'instruction de mercenaires. La Convention a été présentée à l'Assemblée générale aux fins de signature et de ratification en décembre 1989. La Convention adopte une définition bien plus précise que celle du Protocole additionnel I. Ainsi, le recrutement, l'utilisation, le financement et l'instruction des mercenaires deviennent des infractions. Mais, bien qu'une telle définition ne constitue pas un problème en soi, c'est la question du contrôle des activités des individus engagés dans des activités mercenaires qui se pose. Nous reviendrons sur le projet de Convention internationale contre le recrutement, l'utilisation, le financement et l'instruction de mercenaires. En effet, il nous faut en premier lieu examiner les points liés aux problèmes d'une construction d'une définition juridique adéquate du mercenaire.

Le protocole additionnel I fait toujours référence à la motivation lorsqu'est faite la distinction entre mercenaires et d'autres types de combattants. Les Etats occidentaux ont été extrêmement critiques sur ce point. La motivation des mercenaires s'explique par l'argent. Ainsi, comme l'indique Abraham, «un mercenaire est motivé [...] 
essentiellement par le désir d'un enrichissement personnel $\aleph^{33}$. Toutefois, le rapport Diplock qui a été publié en 1976 à la suite de l'implication de mercenaires britanniques en Angola - rappelle qu'il est virtuellement impossible de déterminer exactement les motivations des combattants : "[...] toute définition des mercenaires qui requerrait un indice positif de motivation serait [...] inopérante, ou tellement hasardeuse dans son application entre deux cas individuels identiques qu'elle en serait inapplicable. Nous pensons que les mercenaires ne peuvent être définis qu'en référence à ce qu'ils font, et non en référence à pourquoi ils le font $\aleph^{34}$.

Alors que la motivation ne devrait pas seulement comprendre les éléments de définition, les objections à son utilisation comme partie de la définition ne sont pas plus convaincantes. En droit interne, les distinctions fondées sur la motivation sont régulièrement faites. Il semble qu'il n'y ait pas plus de raison, à la lumière du Protocole additionnel I, de rejeter la nécessité d'un faisceau d'indices pour établir la motivation. Il est très difficile d'obtenir les indices nécessaires pour pouvoir prouver les cinq premiers éléments consignés dans la liste de Taulbee (voir supra) puisque cela nécessiterait d'avoir accès aux archives de la partie opposée au conflit, une situation qu'il est peu probable de voir se produire.

Taulbee identifie six conditions relatives au problème de la distinction entre les mercenaires et les autres volontaires étrangers ${ }^{35}$ :

Si une distinction peut être faite entre des non-citoyens et des non-citoyens résidents;

$\mathrm{Si}$ « mercenaire » comprend tous ceux qui répondent à certains examens opérationnels, ou si des actions évidentes et directement en rapport avec les hostilités sont nécessaires ;

Si les forces privées extérieures et les troupes nationales doivent être considérées comme différentes des Etats tiers ;

Si les individus recrutés pour un conflit spécifique doivent être distingués de ceux recrutés sous d'autres circonstances ;

Si les motifs doivent être définis au travers de tests objectifs ;

Si une distinction juridique doit être faite entre les mouvements légitimes et nonlégitimes de libération nationale.

Cependant, le problème d'obtention des preuves nécessaires à des poursuites existe réellement, compte tenu des statuts qui prévalent dans les juridictions extraterritoriales au détriment des juridictions internes, et ainsi, il existe peu de raisons d'objecter à des questions de ce type ${ }^{36}$. Plus importante est la question des indemnités qui est essentielle dans l'établissement de distinctions dans les motivations. Pour les Etats africains, les indemnités en tant que moyen d'enrichissement personnel distinguent les mercenaires des non-ressortissants non-résidents qui se portent volontaires pour venir en aide aux mouvements de libération légitimes. Si l'attirance pour un enrichissement personnel est retirée de la définition alors la définition perd toute substance. Le terme de mercenaire décrirait ainsi simplement tous les nonrésidents non-ressortissants qui auraient choisi de se battre pour une raison quelconque, y compris politique ou religieuse. Ceci apparait alors comme un test important en vue de déterminer le statut des combattants. Bien que pour les Etats la question de savoir comment apporter des compensations autres que financières demeure confuse. 
L'origine géographique sert aussi à définir le terme de mercenaire. Le mercenaire n'est ni le ressortissant d'un Etat prenant part au conflit, ni un résident d'un territoire contrôlé par une partie en conflit. Au contraire, il s'agit plus généralement « de bandes de soldats professionnels [...] temporairement unis, placés sous l'autorité de leaders à forte personnalité, se battant pour l'argent et [le butin de guerre], mais pas entièrement indifférents aux principes d'honneur et de légalité, ou aux intérêts de leur pays d'origine ${ }^{37}$. L'idée d'utiliser la notion d'origine dans le droit international afin de mieux déterminer la répartition des droits et obligations d'un ressortissant n'est pas forcément le moyen le plus efficace de relier un individu à un territoire précis. Des non-ressortissants résident souvent dans des territoires où éclatent des conflits. A ce titre, même en tant que nonressortissants, ils peuvent estimer avoir des intérêts substantiels à protéger. Une telle personne pourrait très bien disposer d'importantes compétences, et des connaissances locales, que l'une des parties en conflit serait prête à payer au prix fort. Ainsi, dans la Convention de $\mathrm{l}^{\prime} \mathrm{OUA}^{38}$ et dans le projet de Convention de Luanda ${ }^{39}$, cette variété particulière d'individus peut être considérée comme mercenaire. Leur statut dépend entièrement du camp pour lequel ils choisissent de se battre dans un conflit. Le test critique pourrait consister à savoir si un tel individu s'est opposé aux mouvements de libération ou d'autodétermination ${ }^{40}$.

La définition du mercenaire telle que consignée dans le Protocole additionnel I apporte des réponses conservatrices aux questions mentionnées plus haut. De plus, pour qu'une personne soit réputée mercenaire, elle doit avoir réuni tous les critères de définition ${ }^{41}$. Comme cela a été dit précédemment, toute personne réputée mercenaire n'a pas droit au statut protecteur du combattant et de prisonnier de guerre dont jouit le soldat d'une armée régulière. L'objectif du Protocole additionnel I et II est de s'assurer que les participants aux combats contre la domination coloniale, les régimes racistes ou la domination étrangère se voient conférer un statut protecteur, alors que les dispositions s'appliquent à l'utilisation des mercenaires en général. L'engagement de mercenaires dans des mouvements de libération nationale n'est pas, une fois encore, traité de façon isolée. Ce point est particulièrement intéressant à soulever car la définition dans le Protocole additionnel I ne reflète pas l'idée générale contenue dans les précédentes résolutions de l'ONU, la Convention de l'OUA et le projet de Convention de Luanda.

La définition du Protocole additionnel I représente un compromis dans le sens où les membres de l'Organisation de coopération et de développement économiques (OCDE) ont énergiquement soutenu que la responsabilité pénale ne pouvait être engagée que si des actes de guerre précis étaient commis ${ }^{42}$; alors que la majorité des autres pays voulaient une définition plus large du mercenaire afin d'y inclure l'idée que le statut de mercenaire à lui seul constituait un crime. Les Etats qui recherchaient la définition la plus complète pointèrent du doigt le fait qu'un enrôlement volontaire dans une armée mercenaire signifie une intention, et qu'à ce titre l'engagement devrait automatiquement signifier la responsabilité pénale de l'individu. Ainsi, mettre l'accent sur l'acte volontaire que constitue l'engagement dans une armée mercenaire signifie que le facteur déterminant du statut de mercenaire comporte une responsabilité supplémentaire. Toute personne menant des actions listées dans les projets de convention (dont la Convention internationale contre le recrutement, l'utilisation, le financement et l'instruction de mercenaires) peut être l'objet de poursuites, ce qui constitue une dissuasion supplémentaire à l'encontre d'un tel individu. 
Les Etats africains ont aussi essayé d'étendre la définition de mercenaire afin d'y adjoindre les mercenaires qui ne seraient pas couverts par la définition du Protocole additionnel I. Un certain nombre d'Etats ont avancé le fait que le protocole additionnel I ne couvre que les mercenaires engagés dans des conflits armés de nature internationale. Le Protocole ne couvre pas les guerres civiles alors même que les activités mercenaires y sont plus importantes. Aussi, toute définition utile du mercenariat doit comprendre tout autant les situations de guerre infra-étatiques qu'internationales.

Les délégués des Etats occidentaux ont accepté que le projet nigérian de Convention pour l'élimination des mercenaires en Afrique devait intégrer les situations qui ne sont pas couvertes par le Protocole additionnel I. En s'accordant sur cette position, les Etats occidentaux ont affirmé que deux conditions principales doivent être requises. Tout d'abord que la définition consignée dans le Protocole additionnel I ne s'applique pas seulement aux situations de conflit armé international ${ }^{43}$, ensuite que toute définition élaborée dans des conventions ultérieures doit rester conforme à la définition du Protocole additionnel I afin de maintenir l'intégrité du régime établi dans les Protocoles additionnels I et II. Tout autre cas qui ne tomberait pas dans la catégorie des conflits armés internationaux devrait être contenu dans une formule qui suive la ligne adoptée par la définition du Protocole additionnel I ${ }^{44}$. Les Etats occidentaux avancent que la manière qui conviendrait le mieux pour régler cette question, serait d'interdire certaines pratiques et activités dans un contexte parfaitement défini. Taulbee ajoute ${ }^{45}$ que ces représentants devraient élargir les éventualités qui pourraient s'appliquer à la définition du Protocole additionnel I, plutôt que d'étendre les types d'activité et de personne précisément inclus dans la définition.

Afin de réconcilier des points de vue différents entre les Etats membres de l'ONU, le résultat de ces négociations a été la présentation le 4 décembre 1989 de la Convention internationale contre le recrutement, l'utilisation, le financement et l'instruction de mercenaires ${ }^{46}$. Les articles présents dans la Convention contiennent pour beaucoup les idées défendues par les Etats africains. La Convention diffère toutefois des dispositions conservatrices contenues dans le Protocole additionnel I. L'approche adoptée est celle des délits précis afin d'élargir la définition du mercenariat à des situations qui dépassent le cadre du conflit armé international. Ainsi, les faits mentionnés dans le titre de la Convention deviennent identiques à une participation directe dans le sens des dispositions prévues dans le Protocole additionnel I.

La Convention internationale contre le recrutement, l'utilisation, le financement et l'instruction de mercenaires n'est pas exempte de toute critique. Abraham désigne trois domaines pour lesquels la Convention est considérée comme problématique ${ }^{47}$ :

c'est seulement lorsque le crime de mercenariat est perpétré au sein des frontières d'un Etat ou par le ressortissant d'un Etat, que cet Etat se voit accorder la juridiction pour juger de ce crime; en cas de conflit, la Convention refuse à l'Etat lésé le droit de poursuivre l'Etat fautif ;

La Convention ne prévoit aucun mécanisme de contrôle sur ces dispositions, plaçant ainsi la responsabilité sur les Etats-membres eux-mêmes.

Depuis 1989, date à laquelle la Convention internationale contre le recrutement, l'utilisation, le financement et l'instruction de mercenaires a été signée, l'Assemblée générale des Nations unies a continué à passer des résolutions relatives aux activités des mercenaires. Ces résolutions ont reflété en général la nature restrictive de l'interdiction de l'utilisation de mercenaires, de même que les inquiétudes 
traditionnelles exprimées par la communauté internationale vis-à-vis de l'activité d'individus engagés dans le mercenariat ${ }^{48}$ tout en s'intéressant aux actions des mercenaires dans de situations très différentes. Celles-ci comprennent la déstabilisation des Etats voisins, la préparation d'un coup d'Etat dans un petit pays, des actions contre des mouvements de libération nationale dans leur lutte pour l'indépendance, et la violation des droits de l'homme ${ }^{49}$. Le sens de ces résolutions est de souligner le fait que les actions de mercenaires contreviennent aux principes fondamentaux du droit international, y compris la non-ingérence dans les affaires intérieures des Etats, l'intégrité territoriale et l'indépendance ${ }^{50}$. Ce type de mercenariat est décrit par Marie-France Major comme « un acte international illégal » ${ }^{51}$.

Encore une fois, il n'y a pas d'interdiction totale quant à l'utilisation des mercenaires dans le droit international coutumier. Ces conventions présentées par la Communauté internationale se sont concentrées sur l'interdiction d'activités mercenaires attentatoires à la souveraineté des Etats légitimes, à la suppression des mouvements de libération nationale ou à l'autodétermination. Ces activités entreprises par des Compagnies militaires privées en Afrique et en d'autres endroits du monde entrent dans la majorité des cas en dehors de cette caractérisation. Nous reviendrons plus tard à cette question. Mais brièvement, elles n'ont pas été considérées comme défiant la souveraineté des Etats, s'opposant aux mouvements de libération nationale ou comme étant dirigées contre des mouvements d'autodétermination. Comme l'affirme Zarate, en Afrique s'est développée «la claire distinction entre le soutien étranger aux régimes africains légitimes et les tentatives mercenaires individualisées de faire des ravages dans une région $»^{52}$.

L'effort continu pour une condamnation du mercenariat au travers des différentes institutions de l'ONU est considéré par certains (dont l'objectif est d'interpréter cette part du droit international relative aux activités mercenaires) comme constituant la preuve d'une règle, à savoir celle selon laquelle « les Etats ont une obligation légale qui va au-delà des obligations traditionnelles du droit international qui consiste en un contrôle du recrutement de leurs propres ressortissants lorsqu'une situation de menace contre la paix et la sécurité existe ${ }^{53}$. Ceux qui reprennent cet argument ne reconnaissent pas qu'une telle condamnation et de telles résolutions soient dirigées contre des conflits bien précis qui ont vu des mercenaires poser une menace potentielle à la paix et à la sécurité internationales. Ainsi, ces condamnations et résolutions ne constituent pas nécessairement une opposition totale à l'utilisation de mercenaires.

En allant plus loin, même là où la condamnation et les résolutions exprimées ont été plus générales dans leur portée et leur sens, les déclarations ne vont pas à l'appui des normes internationales coutumières. Dans ce domaine, l'Assemblée générale ne se voit pas conférer par la Charte de l'ONU le pouvoir de créer, modifier ou abroger les règles $\mathrm{du}$ droit international. Aussi, la prolifération ces quatre dernières décennies de résolutions et d'itératives recommandations sur le mercenariat ne signifient pas la preuve d'une volonté de s'engager de la part des Etats, ni d'une opinio juris qui seraient nécessaires pour qu'une telle pratique devienne une règle du droit international. Tout ce que ces résolutions et recommandations issues de l'Assemblée générale ou d'organisations régionales (dont l'OUA) peuvent apporter, serait de contribuer à un éventuel établissement d'une règle coutumière à venir du droit international. Et audelà de cette question, se pose le problème de la ratification. Pour que la Convention internationale contre le recrutement, l'utilisation, le financement et l'instruction de 
mercenaires constitue une règle du droit international, elle doit être ratifiée. Or seuls seize Etats ont signé la Convention. Parmi ceux-ci, trois Etats ont loué ou même directement employé des compagnies militaires privées (Angola, Congo et Nigeria) ${ }^{54}$. La Convention nécessite vingt-deux ratifications pour qu'elle puisse entrer en vigueur. C'est pourquoi, de fait, l'impact juridique de la Convention en est fortement réduit, ce qui donne ainsi un poids supplémentaire à ceux qui tentent de saper les arguments des tenants du droit international coutumier ${ }^{55}$.

Les mercenaires et la responsabilité des Etats

La notion de responsabilité des Etats a, par le passé, été liée à l'idée de souveraineté territoriale. Alors qu'un Etat peut se prévaloir du droit à l'indépendance et à l'intégrité territoriale comme tout autre Etat membre de la communauté internationale, détenir un tel droit exige de la part des autres Etats de respecter et protéger ce droit lorsqu'il s'applique aux autres. Taulbee remarque qu'il y a deux domaines de responsabilité qu'un Etat reconnaît quand il viole le droit international. D'abord les pertes et dommages subis, et ensuite la responsabilité des Etats ${ }^{56}$. La validité de ces propositions a rarement été mise en cause ${ }^{57}$.

L'objet de ce débat tient d'abord au lien fait entre non respect des obligations et responsabilité. La discorde tient à la réalisation d'une norme de comportement requise par les Etats dans un contexte d'extension de l'autorité juridique d'un Etat pour protéger le droit des autres Etats. La norme de comportement d'un Etat vis-à-vis du contrôle des activités mercenaires et des compagnies militaires privées ne constitue qu'une partie du débat en cours. La conception de l'Etat suggère que l'Etat soit à même d'assurer certaines fonctions. Une de ces fonctions (qui est réputée se trouver dans l'éventail des fonctions qu'un gouvernement doit réaliser, depuis qu'il a l'autorité absolue sur son propre territoire) est d'assurer l'ordre dans les activités de ses propres ressortissants au sein de ses propres frontières ${ }^{58}$. García-Mora affirme que «l'Etat qui ne peut empêcher un acte violent contre un autre Etat a violé l'obligation internationale de préserver l'ordre mondial " ${ }^{59}$. De plus, quand bien même " un Etat a usé de tous les moyens en son pouvoir pour empêcher toute action illégale contre un Etat étranger, mais n'a pu remédier à la situation, il a manqué à ses obligations et demeure par conséquent responsable $\aleph^{60}$. La conséquence pour les Etats d'avoir manqué à l'exercice de leurs obligations internationales vis-à-vis des autres Etats membres est qu'il leur est imposé, par l'intermédiaire d'un tribunal arbitral ou d'une cour, une règle absolue de responsabilité. Par le passé, des normes relatives ont été imposées et ont cherché à comprendre deux éléments : connaissance et capacité. Le fait que soit portée à la connaissance d'un Etat une action violente commise, ou sur le point d'être commise, n'est pas un élément suffisant pour engager la responsabilité de l'Etat. Le droit international traditionnel demande aux Etats de démontrer leurs bonnes intentions en développant tous les efforts raisonnables pour protéger les Etats membres d'actes d'agression commis par leurs ressortissants et de punir les coupables.

La question de savoir dans quelle mesure un Etat peut ou devrait être tenu pour responsable de ses ressortissants engagés dans des activités mercenaires est extrêmement délicate. C'est d'autant plus le cas aujourd'hui qu'il y a convergence des motivations dans la définition du mercenaire, tant dans le Protocole additionnel, que dans la Convention internationale contre le recrutement, l'utilisation, le financement et l'instruction de mercenaires. Sur ce point, les deux conventions ignorent la responsabilité des Etats quant aux actions menées par leurs ressortissants. En effet, 
comme le souligne Abraham, «ces normes péremptoires qui existent de fait ont évité aux Etats de se voir imputer une responsabilité pour les actions de leurs citoyens ${ }^{61}$. Cette répugnance est partiellement due «à la peur que la communauté internationale entretient des mercenaires... en ce sens où ils sont totalement indépendants de toute obligation construite au sein du système de l'Etat nation ${ }^{62}$. Ainsi, est avancée l'idée que les Etats ont peu de moyens à leur disposition pour pouvoir empêcher leurs ressortissants de mener des activités mercenaires et ne devraient, par conséquent, pas être tenus pour responsables des ressortissants qui entreprennent de telles activités. Un Etat qui accepte ce point de vue ignore ses propres obligations à faire respecter les principes d'intégrité territoriale et d'indépendance politique des autres Etats composants la communauté internationale, et néglige ainsi sa responsabilité à s'assurer que ses propres ressortissants se comportent d'une manière qui ne soit pas attentatoire à ces obligations. Si ce problème doit être abordé, alors devra être intégré au droit international le pouvoir d'attribuer la responsabilité des Etats pour les actions commises par leurs ressortissants. Une telle responsabilité naîtrait d'une reconnaissance par les Etats, du fait de leur appartenance à la communauté internationale, de leur nouvelle responsabilité d'Etats modernes.

Enfin, le cœur du débat n'est pas de savoir si un Etat a des responsabilités nées des obligations envers la communauté internationale (ce point n'est pas disputé), mais de savoir jusqu'où s'étend cette responsabilité. Le droit a par le passé tenu pour irraisonnable de tenter d'imputer une responsabilité à l'Etat là où il y a eu tentative satisfaisante de mettre fin aux actions menées par ses propres ressortissants et dirigées contre un autre Etat. Ainsi, si un Etat mis en cause ne peut avoir une connaissance préalable de ces actions, il n'a que très peu de moyens pour les empêcher, et c'est ainsi que la communauté internationale n'a pas estimé approprié de punir l'Etat en cause pour des faits commis par ses ressortissants. De plus, de nombreux Etats, et particulièrement les moins développés, ne disposent pas des ressources nécessaires pour pouvoir contrôler les faits et gestes de leurs ressortissants. Il n'est donc pas surprenant que la communauté internationale ait soutenu les tentatives de certains Etats de mettre en place des mesures administratives et législatives afin de contrôler les actions de leurs ressortissants. Aussi, l'application de telles mesures législatives estelle possible pour la plupart des Etats de la communauté internationale, y compris les moins développés.

Le moteur de cette législation contre les mercenaires est issu des pays du Tiers monde. Les Etats africains en particulier ont cherché à négocier une convention multilatérale relative à l'utilisation de mercenaires dans les conflits armés de par le monde. Ce n'est pas très surprenant. Si nous traçons l'histoire de l'implication des mercenaires dans des conflits armés depuis les années 1960, nous voyons que la plupart prennent place sur le continent africain. Il en résulte dans les commentaires les plus récents à ce sujet qu'il y a désormais un besoin de renforcer les lois existantes relatives à tous les aspects du mercenariat, de même que de rendre les Etats plus responsables encore des actions engagées par leurs ressortissants. La Convention internationale contre le recrutement, l'utilisation, le financement et l'instruction de mercenaires traite de l'interdiction des activités des mercenaires soit en tant qu'individus, soit en tant que groupes; mais elle n'a pu faire porter la responsabilité aux Etats pour leurs ressortissants qui s'engagent dans une activité mercenaire. Aussi, l'impact que la Convention a eu pour empêcher les mercenaires de poursuivre leur commerce a été manifestement réduit. Le sens de la Convention peut ainsi être apprécié en des termes plus symboliques que substantiels. 
Les inquiétudes et les efforts actuels pourraient être aussi dirigés contre un phénomène qui est en réalité transitoire par nature.

Les compagnies militaires privées et les entreprises mercenaires : une distinction juridique

Le débat jusqu'à maintenant portait sur les problèmes liés à la construction d'une définition juridique du mercenariat, et à la responsabilité des Etats pour empêcher leurs ressortissants de prendre ainsi part à des activités mercenaires. Aussi, nous avons vu combien il est difficile de donner une définition juridique précise du mercenariat qui puisse recouvrir toutes les possibilités d'activité mercenaire, qui soit acceptée par les Etats et à laquelle les Etats soient enclins à souscrire. Devant une tâche aussi difficile, toute tentative d'étiqueter ceux qui travaillent pour des compagnies militaires privées de mercenaires, ne fait que compliquer plus encore la question de la définition. A ce sujet, David Shearer avance les principales difficultés d'application du critère contenu à l'article 47 relatif au personnel des compagnies militaires. Shearer distingue quatre zones principales de friction ${ }^{63}$ :

Au sous-paragraphe (a), il est précisé que le recrutement doit être effectué spécifiquement pour un conflit armé précis. Comme beaucoup de ceux qui travaillent dans les compagnies militaires et sont employés sur la base d'un contrat de longue durée, ils peuvent justement ne pas être considérés comme mercenaires.

La nécessité que les mercenaires prennent directement part aux hostilités est requise au sous-paragraphe (b), et exclurait ainsi tous les individus travaillant comme conseillers ou techniciens militaires étrangers.

Le besoin d'établir un "désir d'enrichissement personnel » au sous-paragraphe (c) est difficile à prouver parce qu'il introduit un élément psychologique, une motivation. Cet aspect a longuement été discuté plus haut.

Au sous-paragraphe (c) un membre d'une force armée partie prenante d'un conflit ne peut pas être pris pour un mercenaire. Ainsi, en devenant un membre de forces armées locales, les combattants ayant signé un contrat évitent l'appellation de mercenaire contenue au sous-paragraphe (e). Sandline, dans son contrat avec le gouvernement de Papouasie Nouvelle Guinée, avait fait figurer l'appellation de "gendarmes spéciaux " ${ }^{64}$ pour ses employés, sans aucun doute pour renforcer la distinction.

Les employés de compagnies militaires privées, dont le travail est d'apporter un soutien militaire, sont fréquemment accusés d'être des mercenaires (bien qu'ils travaillent sous couvert d'une entreprise vénérable pour un travail honorable) et donc d'opérer illégalement. Bien que problématique, établir le statut juridique des compagnies militaires privées est très important, puisque cela a des implications sur la conduite des employés des compagnies militaires privées impliquées dans la promotion de la sécurité internationale en général. Bien que de telles exclusions, comme le précise Shearer, soient d'ordre technique (ou chaque paragraphe et sous-paragraphe demeure suffisamment ouvert afin de rendre possible une remise en cause de leur sens), prises ensemble, elles rendent l'article 47 totalement inopérant, tant pour les mercenaires que pour les compagnies militaires privées ${ }^{65}$. Cela est naturellement intentionnel. Les Etats africains, dans la construction juridique de la définition du mercenariat, ont été très attentifs à s'assurer que la définition soit suffisamment ouverte pour qu'elle soit sujette à désaccord, et qu'elle leur donne l'opportunité d'employer la force militaire qu'ils jugeront nécessaire pour se maintenir au pouvoir. 
Devrions-nous alors définir ceux qui travaillent pour les compagnies militaires privées comme mercenaires? Il s'agit ici d'une question plus politique que juridique, car les raisons de décider de l'un ou de l'autre seront toujours conduites par la politique. Ceux qui s'opposent à toute implication militaire privée dans la guerre pensent que ceux qui travaillent pour des compagnies militaires privées devraient être tenus pour mercenaires uniquement, et qu'aucune autre appellation qui puisse cacher ce fait ne devrait leur être attachée. Pour ce groupe, les armées étatiques, ou les mouvements de libération nationale représentant des communautés politiques, sont les seules et uniques forces à être autorisées à mener la guerre. La guerre est le domaine de ces deux groupes, et de fait, ceux-ci sont responsables de la protection physique des communautés qu'ils représentent. D'un autre côté, les compagnies militaires privées sont souvent considérées comme représentant les intérêts économiques d'un groupe minoritaire, habituellement les intérêts occidentaux, qui n'ont rien en commun avec ceux chargés de la protection. Ceux qui travaillent pour le compte de compagnies militaires privées devraient donc être appelés mercenaires, en ce sens où ils représentent une organisation voulant vendre des compétences militaires au plus offrant, et pour qui la cause importe peu. Les seules exceptions concernent les étrangers qui choisissent de se battre en raison de leurs convictions politiques. De tels individus ne perçoivent aucun bénéfice économique de leur participation au combat, à la différence de ceux travaillant pour les compagnies militaires privées, et sont donc ainsi décrits comme volontaires.

Ceux qui sont opposés à ce point de vue pointent du doigt les programmes politiques de ceux qui décrivent les compagnies militaires privées comme représentant rien moins que des entreprises mercenaires. C'est particulièrement le discours des forces armées qui se considèrent comme victimes du succès des compagnies militaires privées, qu'elles soient ou non reconnues légitimement par leur peuple. Finalement, la question de savoir si une personne ayant signé un contrat pour apporter une assistance militaire à une armée étrangère est un mercenaire, un volontaire ou un conseiller militaire, est laissée à l'appréciation de la conscience de chacun, influencé par ses propres croyances et valeurs politiques. Considérons la question de l'encadrement des compagnies militaires privées : la communauté internationale est à ce point hésitante quant à ses intentions de rendre les actions des compagnies militaires privées illégales qu'elle ne laisse pas d'autre alternative que de les contrôler au travers d'une réglementation.

Le problème de la définition est clairement lié aux problèmes politiques associés à la répugnance des Etats à exclure toute utilisation de mercenaires. Le résultat a donné naissance à des tensions entre les Etats africains en particulier, et l'Occident, dont l'Europe principalement. Alors que les leaders africains ont mené la lutte pour réformer le droit international relatif aux mercenaires, l'Occident est resté prudent. De telles tensions se remarquent dans la définition du Protocole additionnel I. En effet, alors que les pays de l'OCDE estiment la responsabilité pénale comme étant engagée à la suite d'actes de guerre précis, la majorité des Etats a cherché à inclure l'idée que le statut seul suffit pour constituer un acte criminel. Les tentatives d'apaisement de ces tensions se révèlent délicates.

L'efficacité de l'actuelle législation internationale sur les mercenaires est très limitée ${ }^{66}$. Mais cette faiblesse de la loi est manifestement le fruit d'une volonté partagée. Les gouvernements africains estiment qu'il n'y a aucune raison de se couper l'accès à une potentielle source d'expertise militaire. Finalement, tout ce que la communauté 
internationale a voulu faire ces cinq dernières décennies c'est se débarrasser des mercenaires indépendants qu'elle souhaite voir hors de certains conflits. Et c'est particulièrement le cas des gouvernements africains qui ont été les cibles principales de ces mercenaires indépendants, et qui le sont toujours, par ailleurs.

Aussi, le droit international ne fait-il pas mention explicite des compagnies militaires privées. Ce n'est pas surprenant compte tenu de l'apparition récente de ces organisations sur la scène internationale. Le problème est de savoir si, à l'avenir, les gouvernements autoriseront les compagnies militaires privées à s'engager activement en leur nom dans des guerres civiles, marquant l'échec d'une séparation légale qui pourrait voir les employés des compagnies militaires privées endosser un statut légal de combattant mais dont ce même statut pourrait être nié par la partie adverse et pourrait mener alors à de terribles conséquences pour les employés qui auraient le malheur d'être capturés. Dans ce domaine, le droit international a deux rôles majeurs à jouer si les compagnies militaires privées doivent agir un jour pour le compte de la communauté internationale. D'abord, le droit international doit protéger le statut de combattant des employés des compagnies militaires privées activement engagés dans le conflit. Ensuite, il doit permettre aux compagnies militaires privées de poursuivre leur activité légalement tout en interdisant toute activité aux mercenaires classiques. Il ne s'agit pas là de tâches faciles à réaliser, mais il le faut si les compagnies militaires privées doivent agir pour le compte de la communauté internationale.

\section{NOTES}

1. Article traduit par Nicolas Wuest-Famôse.

2. . Cité dans J. C. Zarate, «The Emergence of a New Dog of War : Private International Security Companies, International Law, and the New World Order », Stanford Journal of International Law, 1998, $\mathrm{N}^{\circ} 34$, p. 125.

3. . A.F. Musah et J.K. Fayemi (sld.), Mercenaries : An African Security Dilemma, Londres, Puto Press, 2000, pp. 269-271.

4. . Les attentats du 11 septembre 2001 par des membres du réseau al-Qaida contre les tours du World Trade Center à New-York et contre le Pentagone à Washington auront une importance majeure sur ce sujet.

5. . Pour une discussion plus générale sur l'usage des mercenaires hors d'Europe, voir R. Smith, Mercenaries and Mandarins, New-York, KTO Press, 1978.

6. . L. Oppenheim, International Law, (sld. H. Lauterpacht), Londres, Longmas Green \& Co, 7ème édition, 1952, p. 703.

7. . Charte des Nations unies, Article $2 \S 4$, « Les Membres de l'Organisation s'abstiennent, dans leurs relations internationales, de recourir à la menace ou à l'emploi de la force, soit contre l'intégrité territoriale ou l'indépendance politique de tout Etat, soit de toute autre manière incompatible avec les buts des Nations Unies ».

8. . Application de la Résolution de l'Assemblée générale sur le droit à l'indépendance des pays et des peuples coloniaux, Résolution de l'Assemblée générale 2465, 23 UN GAOR Supplément ( $\left.\mathrm{N}^{\circ} 18\right)$, Document ONU A/7218 (1968). 
9. . Les résolutions de l'Assemblée générale de l'ONU ne sont pas transposables directement dans le droit international, ni même les droits nationaux. Au contraire, elles sont comprises comme représentant l'expression de la volonté collective des membres de l'Organisation.

10. . J.L. Taulbee, « Mercenaries and Contemporary International Law », California Western International Law Journal, America, California Western School of Law, 1985, vol. $15, \mathrm{~N}^{\circ} 2$, p. 346.

11. . OUA Convention sur l'Elimination des mercenaires en Afrique, OUA Document CM/433/Rev. L., Annex 1 (1972). La Convention consiste en un law making treaty qui fixe des règles d'application générale, impose des obligations sur les Etats afin d'introduire une législation conforme au cadre de la Convention. La Convention nécessite la signature de 10 Etats pour qu'elle puisse être ratifiée. Jusqu'à présent 22 Etats membres ont signé la Convention, ce qui autorise sa ratification. Elle s'applique uniquement aux Etats membres de l'OUA. Pour plus de détails sur la Convention, lire A. F. Musah, K. Fayemi, op. cit., Appendix V, pp. 286-288.

12. . OUA Convention pour l'élimination des mercenaires en Afrique, article 2. Fn 10.

13. . A.F. Musah, .K. Fayemi (sld.), op. cit., p. 286.

14. . OUA Convention pour l'Elimination des mercenaires en Afrique, article 2,3. Fn 10.

15. OUA Convention pour l'Elimination des mercenaires en Afrique, article 6,7. Fn 10.

16. . A.F. Musah, .K. Fayemi (sld.), op. cit., p. 286.

17. . J. Herbst, «The regulation of private security force ", in The Privatisation of Security in Africa, South Africa, South African Institute of Internationl Affairs, p. 115.

18. . Pour un compte rendu du procès, voir A. Mockler, The New Mercenaries, Londres, Corgi Boks, 1986, pp. 279-309.

19. . Le projet de Convention de Luanda sur la prévention et la suppression des mercenaires, nommée ci-après projet de Convention de Luanda

http://www.1.umn.edu/humanrts/instree/mercenaryconvention.html 25 juillet 2001.

20. . Voir le Projet de Convention de Luanda.

21. . J. Taulbee, « Myths, Mercenaries and Contemporary Internatioal Law », California Western International Law journal, 1985, Vol. 15, º2, pp. 339-363.

22. . Voir le Projet de Convention de Luanda.

23. . F.J. Hampson, « Mercenaries : Diagnosis Before proscription », Netherlands Yearbook of International Law, 1991, Vol. 22, №3, Pays-Bas, Martinus Nijhoff Publishers, p. 27.

24. . Voir le Projet de Convention de Luanda.

25. . F.J. Hampson, op. cit., p. 27.

26. . Protocole I, Article 47, addition à la Convention de Genève du 12 août 1949, se référant à la protection des victimes de conflits internationaux armés (Protocole 1), UN Doc. A/32/144 Annexe 1 (1977).

27. . A. Roberts, R. Guelff, Documents on the Laws of War, Oxford, Oxford University Press, 2000, p. 485.

28. . K. O'Brien, « Military-Advisory Groups and African Security : Privatized

Peacekeeping ", International Peacekeeping, Londres, Franck Cass, 1998, Vol, 5, pp. 78-105.

29. . A. Mockler, op. cit., pp. 35-36.

30. . J. Taulbee, op. cit., p. 349.

31. . A. Roberts, R. Guelff, Documents on the Laws of War, Oxford, Oxford University

Press, 2000. 
32. . On peut trouver des idéalistes des deux côtés du conflit, comme par exemple ces mercenaires britanniques qui se sont battus en Angola, à l'image de Nick Hall. Hall a prétendu ne pas se battre pour l'argent, mais contre le communisme, même si cela impliquait de se battre sans percevoir de solde dans un pays éloigné de chez soi. Voir $C$. Dempster et D. Tomkins, Firepower, Londres, Corgi, 1998, p. 59.

33. . G. Abraham, « The Contemporary Legal Environment », The Privatisation of Security in Africa, South Africa, South African Institute of International Affairs, 1999, p. 97.

34. . Rapport du Committee of Privy Counsellors chargé d'enquêter sur le recrutement des mercenaires, Cmnd. 6569, p. 2.

35. . J. Taulbee, op. cit., p. 351.

36. . Réunir des preuves dans un pays tiers sur un crime commis par un ressortissant étranger pour que celui-ci puisse être poursuivi dans son propre pays n'est pas unique au mercenariat. Un tel problème se posera sans aucun doute avec les affaires de pédophiles ayant commis leur crime à l'étranger mais poursuivis dans leur propre pays, comme cela se passe maintenant au Royaume-Uni.

37. . A. Mockler, op. cit., pp. 33-34.

38. . Voir fn 12 pour une étude de la Convention de l'OUA.

39. . Le projet de Convention de Luanda est partie intégrante à la Convention de l'OUA pour l'élimination des mercenaires en Afrique, OAU Doc CM/433/Rev. L, Annex I (1972), Appendix II. La Commission internationale d'enquête sur les mercenaires à Luanda, a présenté ce projet en juin 1976. Bien que sa signification soit la même que pour la Convention pour l'élimination des mercenaires en Afrique, elle est finalement partie intégrante à la Convention. Hampson la décrit comme un tract politique masquant un texte juridique. Voir F. J. Hampson, op. cit., p. 28.

40. . W. Burchett, D. Roebuck, The Whores of War : Mercenaries Today, 1977, p. 234.

41. . F.J. Hampson, op. cit., p. 29.

42. . Le point de vue occidental sur cette question est présenté dans le résumé des déclarations du 6ème Comité, par MM. Saint-Martin (Canada) et DeStroop (Australie). Uns 38th Session GAOR C.6 U.N Doc A/C.6/38/SR.23 (1983). Déclaration de M. Font (Espagne). Uns 38th Session GAOR C.6 U.N A/C.6/38/SR.25 (1983).

43. . J. Taulbee, op. cit., p. 355.

44. . Ibid.

45. . Ibid.

46. . F.J. Hampson, op. cit., p. 30. Voir aussi fn 42 pour une liste complète des représentants des gouvernements occidentaux qui ont exprimé un point de vue officiel. Pour plus de détails sur cette Convention, voir :

http://untreaty.un.org/ENGLISH/bible/englishinternetbible/partI/chapterXVIII/ treaty6.asp.

et le document $\mathrm{N}^{\circ} \mathrm{A} / \mathrm{RES} / 44 / 34$.

47. . G. Abraham, op. cit., p. 98.

48. . J.C. Zarate, « The Emergence of a New Dog of war : Private International Secuity Companies, International Law, and the New World Order », Stanford Journal of International Law, 34, 1998, p. 70.

49. . G. Abraham, op. cit., p. 98.

50. . G. Abraham, op. cit., p. 99.

51. . M.-F. Major, « Mercenaries and International Law », Journal of Comparative and International Law, 22, 1992, p. 117. 
52. . J. C. Zarate, op. cit., p. 90.

53. . Ibid., p.133.

54. . G. Abraham, op. cit., p. 100.

55. . En fait, la Convention internationale contre le recrutement, l'utilisation, le financement et l'instruction de mercenaires est entrée en vigueur le 20 octobre 2001 lorsque le vingt-deuxième Etat (le Costa Rica) a déposé les instruments de ratification auprès du secrétaire général de l'ONU. Actuellement, cette convention est ratifiée par vingt-cinq Etats.

56. . J. Taulbee, op. cit., p. 356.

57. . J. Taulbee, op. cit., p. 356.

58. . Ce point particulier est vraiment d'actualité avec la guerre des Etats-Unis contre le terrorisme. Certains Etats, en dépit de leur bonne volonté, n'ont pas la capacité de contrôler parfaitement leur propre territoire.

59. . L'argument mis en avant par García-Mora est très pertinent eu égard aux événements du 11 septembre 2001, et ceci de deux façons. D'abord en quoi les attentats du 11 septembre ont-ils suggéré un lien entre l'Etat afghan et les membres d'al-Qaida au sein de l'Afghanistan? Et ce lien était-il suffisant pour justifier une intervention militaire de l'Amérique?

60. . J. Taulbee, op. cit., p. 357.

61. G. Abraham, op. cit., p. 101.

62. . J. C. Zarate, op. cit., p. 122.

63. . D. Shearer, « Private Armies and Military Intervention », Adelphi Paper 316, Oxford, Oxford University Press, 1998, p. 17/18.

64. . Special Constables.

65. . F.J. Hampson, op. cit., pp. 3-38.

66. . Voir « Report on the Question of the Use of Mecenaries as a means of Violating Human Rights and Impeding the Exercise of the Right of People to Self-determination ", UN Doc. E/CN/4/1997/24, 27 janvier 1998. Part 3, Section B, Current International Legislation and its Limitations, published by the Office of the UN High Commissioner for Human Rights pour un compte rendu détaillé de ce qui est inclus dans cette législation.

\section{INDEX}

Mots-clés : droit international, privatisation de la sécurité

Thèmes : MPRI, Nations Unies (ONU), Union africaine (Organisation de l') 